\title{
Construction of a Hep-2 cell line stably transfected with Livin shRNA
}

\author{
Wang $\mathrm{SL}^{1}$, Deng $\mathrm{WT}^{1}$, Wen $\mathrm{GF}^{1}$, Li CW${ }^{1}$, Zeng $\mathrm{YJ}^{2}$ \\ Department of Otorhinolaryngology Head and Neck Surgery, Shantou City Central Hospital, \\ Shantou, China. yjzeng@bjut.edu.cn
}

\begin{abstract}
OBJECTIVE: The aim of this study was to construct a eukaryotic expression plasmid with a short hairpin RNA (shRNA) targeting Livin in order to obtain a stably transfected Hep-2 cell line with a reduced expression of Livin. METHODS: The shRNA targeting Livin mRNA was designed, and a shRNA plasmid and a negative control plasmid were constructed. After amplification in E. coli, restriction endonuclease digestion and sequence confirmation, the plasmids were transfected into Hep-2 cells using Lipofectamine 2000 . The stably transfected cell line was screened using G418, and inhibition of Livin mRNA and protein levels were detected using real-time PCR and western blotting, respectively.

RESULTS: pGenesil-Livin-shRNA eukaryotic expression plasmid was successfully constructed and identified by sequencing. Green fluorescent protein (GFP) expression was observed in Hep-2 cells transfected with shRNA plasmids by fluorescence microscopy. The expression levels of Livin mRNA and protein decreased significantly in Hep-2 cells transfected with the shRNA recombinant plasmid. The mRNA level was reduced by $47.17 \%$, and the protein level was reduced by $34.25 \%$.

CONCLUSION: The shRNA eukaryotic expression plasmid targeting Livin was successfully constructed, which could significantly inhibit the expression of Livin in laryngeal cancer Hep-2 cells. This provides a basis for future research on the function of Livin in Hep-2 cells, and gene therapy for laryngeal cancer (Fig. 5, Ref. 10). Text in PDF www.elis.sk. KEY WORDS: apoptosis regulatory proteins, cell culture, RNA interference, livin gene.
\end{abstract}

\section{Introduction}

Livin is a member of the anti-apoptotic gene family, and plays an important role in the inhibition of apoptosis. Livin has been shown to be highly expressed in several malignant tumors, such as malignant melanoma, colorectal cancer, leukemia, bladder cancer, lung cancer, and nasopharyngeal cancer (1-6). Specific expression of Livin in tumor tissue suggests that livin may serve as a valuable tumor marker. In recent years, many studies shown that livin and malignant tumor development, invasion and metastasis, chemotherapy resistance have a close relationship.

Laryngeal carcinoma is the most common malignant tumor in Otolaryngology, advanced laryngeal cancer need comprehensive treatment, chemotherapy is one of the important ways of comprehensive treatment program. Patients with laryngeal cancer are unresponsive to chemotherapy, probably due to Livin gene. Therefore, if the drug-resistance caused by Livin could be reversed, it will provide a new avenue for clinical treatment of cancer. According to literature review, there was almost no report about expression of Livin in laryngeal carcinoma,

${ }^{1}$ Department of Otorhinolaryngology Head and Neck Surgery, Shantou City Central Hospital, Shantou, China, and ${ }^{2}$ Biomechanics and Medical Information Institute, Beijing University of Technology, Beijing China.

Address for correspondence: Shuangle Wang, Department of Otorhinolaryngology Head and Neck Surgery, Shantou City Central Hospital, Shantou, China.
In this study, we constructed a shRNA eukaryotic expression vector targeting Livin, transfected it into Hep-2 cells, investigated its inhibitory effect on the expression of Livin mRNA and protein, and screened for an effective targeting site, which could provide a basis for future research on the function of Livin in Hep-2 cells.

\section{Materials and methods}

\section{Reagents}

E. coli strain DH5 $\alpha$ and plasmid pGenesil-1 were purchased from Wuhan Genesil Biotechnology Co., Ltd., China. Oligonucleotides, primers, Lipofectamine 2000 and Opti-MEM I serum-free medium were purchased from Life Technologies, USA. Restriction endonucleases Sal I, BamH I and Hind III, T4 ligase, plasmid extraction kit and quantitative real-time PCR reagents were purchased from Takara Biotechnology (Dalian) Co. Ltd., China. Mouse anti-human Livin monoclonal antibody, rabbit anti-human $\beta$-actin monoclonal antibody, rabbit anti-mouse Livin labeled secondary antibody and western blotting reagents were purchased from $\mathrm{Wu}-$ han Boster Biological Technology Ltd., China. Hep-2 cells were provided by the Department of Virology, Union Hospital, Tongji Medical College, Huazhong University of Science and Technology.

Designing of shRNA sequences and construction of recombinants

The shRNA targeting Livin was designed by RNA interference software based on the Livin cDNA sequence in GenBank and shRNA design principles. 
The negative control sequence (HK) was designed by disrupting a portion of the base pairs. Restriction endonuclease sites Hind III and BamH I were incorporated at the 5' and 3' terminals, respectively, to connect the oligonucleotide with pGenesil-1 vector, and the sequence TTCAAGACG was inserted in the middle to form a hairpin. The DNA template sequence is as follows:

Primer structure: BamH I +Sense+Loop+Antisense+Stop signal + SalI + Hind III

Livin positive sense strand: 5'-GGATCCGTCTGGCCTCCTTCTATGATTCAAGACGTCATAGAAGGAGGCCAGACTTTTTTGTCGACAAGCTT-3', antisense strand:

3'-CCTAGGCAGACCGGAGGAAGATACTAAGTTCTGCAGTATCTTCCTCCGGTCTGAAAAAACAGCTGTTCGAA-5',

HK positive sense strand:

5'-GGATCCACTACCGTTGTTATAGGTGTTCAAGACGCACCTATAACAACGGTAGT TTTTTTGTCGACAAGCTT-3', antisense strand:

3'-CCTAGGTGATGGCAACAATATCCACAAGTTCTGCGTGGATATTGTTGCCATCAAAAAAACAGCTGTTCGAA-5'.

The linearized pGenesil-1 vector was digested by two restriction endonucleases; the target gene was ligated by T4 ligase and transformed into DH5 $\alpha$ competent cells. Kanamycin-resistant colonies were selected and amplified. Plasmid DNA was extracted with the plasmid extraction kit according to instructions. The recombinant plasmids were identified using restriction enzyme Sal I and detected by electrophoresis with $1.5 \%$ agarose gel. The selected plasmids were amplified and sequenced at Shanghai BioAsia Biotechnology Co., Ltd., China. The positive plasmid was named pGenesil-Livin, and the negative plasmid was named pGenesil-HK.

Hep-2 cell culture and stable transfection of shRNA plasmids

Hep-2 cells were cultured in Minimum Essential Eagle's Medium, supplemented with $10 \%$ fetal bovine serum (FBS) at $37^{\circ} \mathrm{C}$ in a $5 \%$ carbon dioxide $\left(\mathrm{CO}_{2}\right)$ atmosphere. Transfections were performed according to instructions for use of Lipofectamine 2000. Stable expression cell lines were obtained by resistance screening with G418 at a concentration of $500 \mu \mathrm{g} / \mathrm{mL}$. The monoclonal cells transfected with positive plasmid were named Hep2-pLivin, and the monoclonal cells transfected with negative control plasmid were named Hep2-pHK.

Detection of mRNA expression in Hep2-pLivin and Hep2-pHK cells by real-time $P C R$

The two groups of cells were digested and collected. Cells were lysed, total RNA was extracted using Trizol reagent, and cDNA was synthesized by reverse transcription. Real-time quantitative PCR primers for Livin and $\beta$-actin were designed using Primer 5.0 software. Livin primers: upstream: 5'-GGCATCTTCAGACTGGACCT-3', downstream: 5'-TCCACAGTGTGCAGGAGACT-3'; $\beta$-actin primers: upstream: 5'-GCAAGAGAGGCATCCTCAC-3', downstream: 5'-CCACACGCAGCTCATTGTAG-3'. Each reaction $(20 \mu \mathrm{L})$ included $10 \mu \mathrm{L}$ reaction mix, $0.4 \mu \mathrm{L}$ upstream primer, $0.4 \mu \mathrm{L}$ downstream primer, $0.4 \mu \mathrm{L}$ Rox, $1.0 \mu \mathrm{L}$ cDNA, and $7.8 \mu \mathrm{L}$
$\mathrm{ddH}_{2} \mathrm{O}$. Reactions were carried out at $95{ }^{\circ} \mathrm{C}$ for $5 \mathrm{~min}$ followed by 40 cycles of $95^{\circ} \mathrm{C}$ for $30 \mathrm{~s}$, and $60^{\circ} \mathrm{C}$ for $30 \mathrm{~s}$. The value of $\Delta \mathrm{CT}$ was plotted as log of the cDNA gradient concentration, and relative quantification was performed by $2^{-\Delta \Delta \mathrm{CT}}$ method. Results were analyzed using the following formula: $\mathrm{mRNA}=2^{-\Delta \Delta \mathrm{CT}}$, $\Delta \Delta C \mathrm{C}=\mathrm{CTLivin}-\mathrm{CT} / \beta$-actin, with $\beta$-actin as a reference. The experiment was repeated three times and averaged.

\section{Detection of Livin protein expression by western blotting}

The two groups of cells were digested and collected. The total protein was isolated. Proteins were separated by electrophoresis with sodium dodecyl sulfate-polyacrylamide gel (SDS-PAGE) and then electrically transferred onto polyvinylidene difluoride (PVDF) membranes. The membranes were incubated with mouse anti-human Livin monoclonal antibody overnight at $4{ }^{\circ} \mathrm{C}$ followed by incubation with enzyme-conjugated rabbit anti-mouse secondary antibodies for $1 \mathrm{~h}$ at room temperature. Protein levels were normalized to $\beta$-actin as a reference. The experiment was repeated three times to observe the changes in expression of the protein.

\section{Statistical analysis}

The data were expressed as the mean \pm standard deviation. Results were analyzed with SPSS 16.0 software. Paired $t$-test was used for statistical analysis between the two groups. $\mathrm{p}<0.05$ was considered to be statistically significant.

\section{Results}

Sequencing of Livin shRNA eukaryotic expression vector

Empty vector was digested with the restriction enzymes and electrophoresed to confirm that the circular plasmid was cut into a large fragment of $4.3 \mathrm{~kb}$ (Fig. 1). Sequencing results confirmed that the shRNA recombinant plasmid was consistent with the target sequence, indicating that the oligonucleotides had annealed with pGenesil-1 vector and the shRNA recombinant plasmid targeting Livin was successfully constructed (Fig. 2).

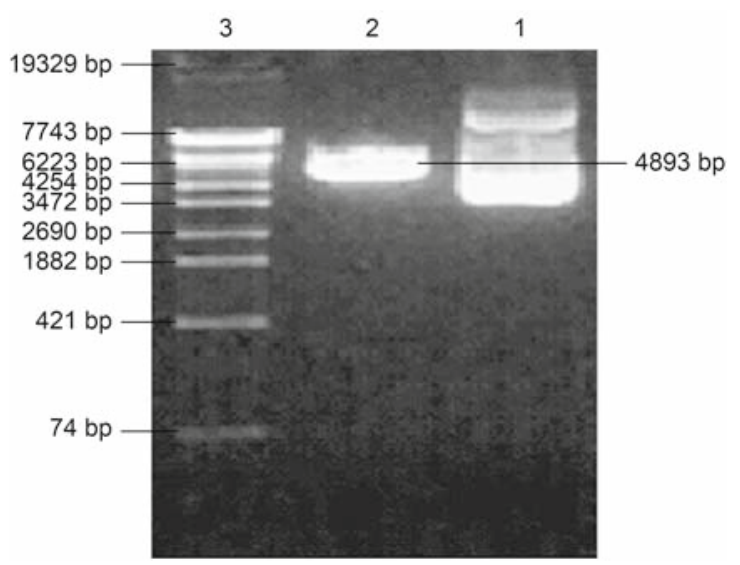

Fig. 1. Electrophoresis map of vectors after restriction enzyme digestion. Lane 1, pGenesil-1 plasmid; lane 2, linearized pGenesil-1 plasmid (digested with BamH I and Hind III); lane 3, marker. 

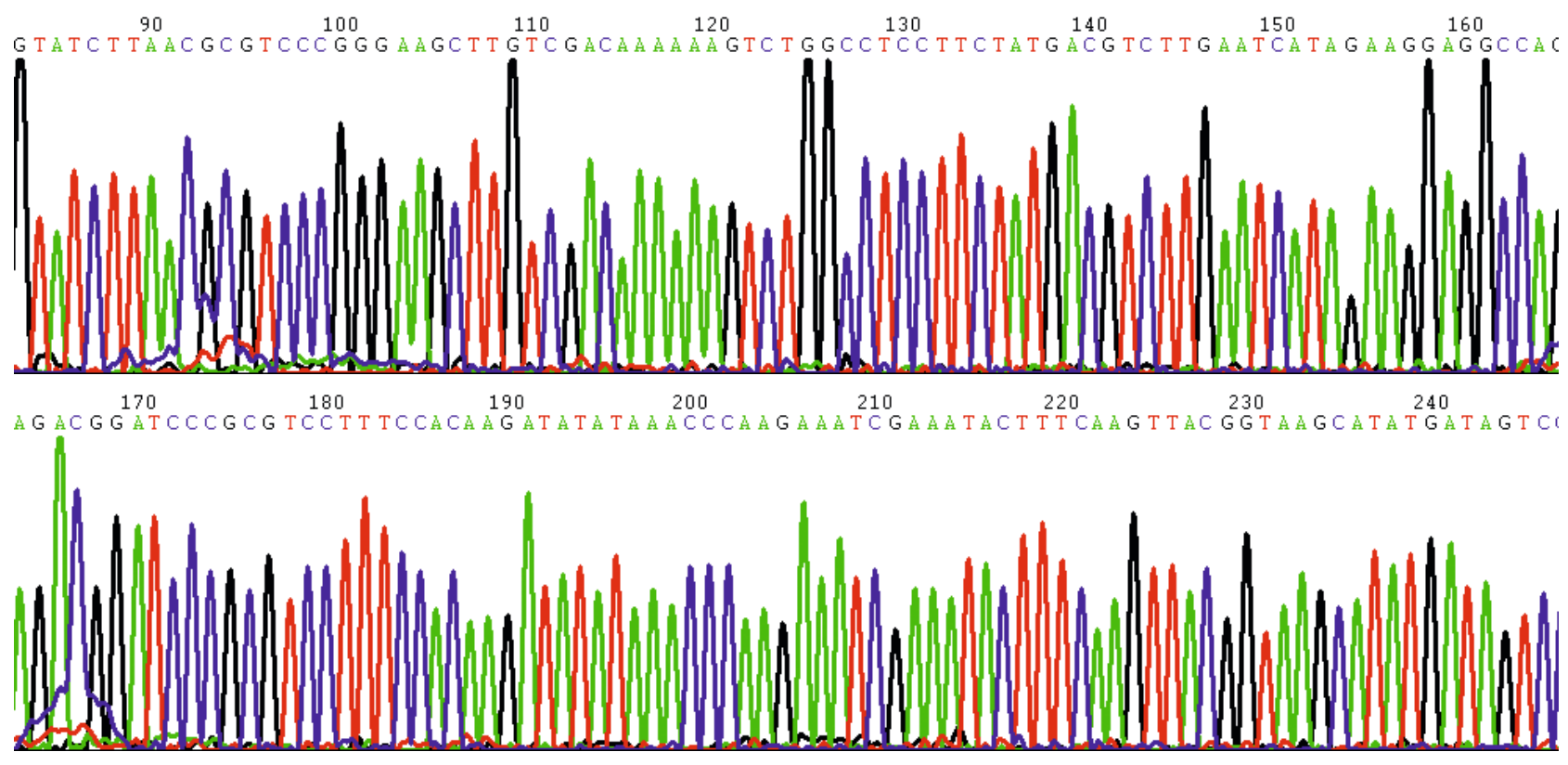

Fig. 2. Sequencing results of pGenesil-1-Livin eukaryotic expression plasmid.
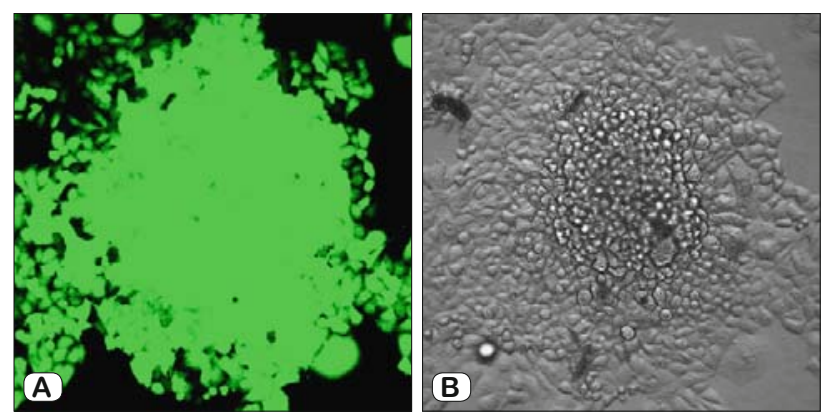

Fig. 3. Hep-2 cell colonies stably transfected with pGenesil-1-LivinshRNA. A: shows that all cells emit bright green fluorescence by fluorescent excitation, $B$ : shows the cell morphology in the same field under a bright light. Cell colonies grew by central outward expansion (200x).
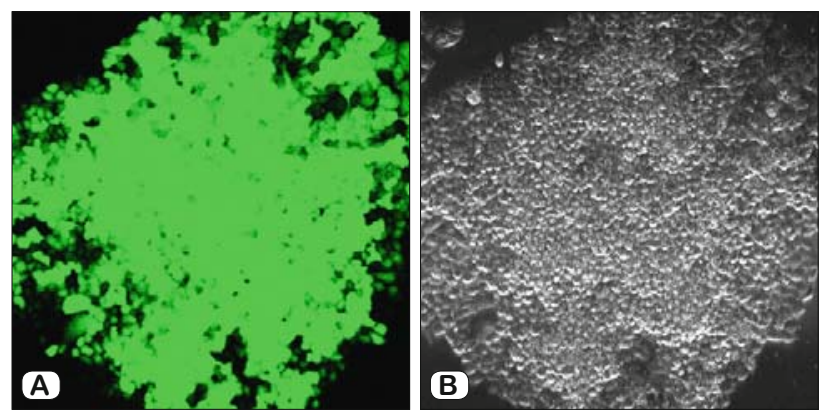

Fig. 4. Hep-2 cell colonies stably transfected with pGenesil-HK-shRNA. A: shows that all cells emit bright green fluorescence by fluorescent excitation, B: shows the cell morphology in the same field under a bright light. Cell colonies grew by central outward expansion (200x).

\section{Visualization of transfected cells by fluorescence microscopy}

Green fluorescence was detected in the cells, which were successfully transfected with pGenesil-Livin-shRNA or negative control
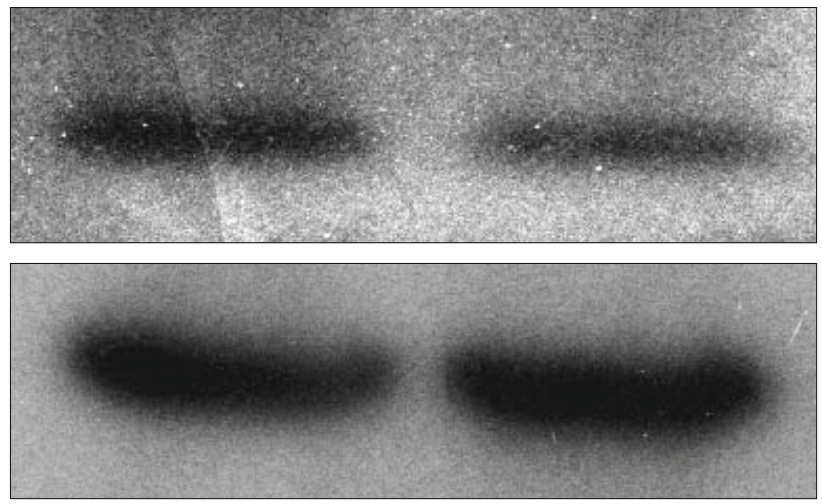

Fig. 5. Relative expression levels of Livin protein in Hep-2 cells stably transfected with Livin shRNA or HK shRNA.

pGenesil-HK-shRNA due to the presence of enhanced green fluorescent protein (EGFP) reporter gene in pGenesil-1 vector, indicating the expression of exogenous genes in transfected cells (Figs 3 and 4).

Effect of Livin-shRNA recombinant plasmid on Livin $m R N A$ expression

Quantitative real-time PCR showed that the relative expression of Livin mRNA in positive plasmid transfected cells was $0.53 \pm$ 0.02 , and the Livin mRNA expression in negative plasmid transfected cells was $100 \%$. Additionally, Livin mRNA expression in laryngeal cancer cells transfected with Livin shRNA was significantly reduced $(\mathrm{t}=-65.24, \mathrm{p}<0.05)$. The inhibition rate was $47.17 \%$.

Effect of Livin-shRNA recombinant plasmid on Livin protein expression

Western blotting results showed that relative expression levels of Livin protein in Hep-2 cells after stable transfection of positive 
plasmid was $0.48 \pm 0.03$, which was significantly lower as compared to cells transfected with the negative plasmid $(0.73 \pm 0.03$, $\mathrm{t}=-17.57, \mathrm{P}<0.05)$. This was consistent with the real-time PCR results, indicating that the expression of Livin in Hep-2 cells after shRNA transfection was significantly reduced, and the inhibition rate was $34.25 \%$ (Fig. 5).

\section{Discussion}

RNA interference technology involves construction of short double-stranded RNAs, which bind to target transcripts and trigger their destruction to specifically inhibit the expression of target proteins (7). The benefits of this technology include high specificity, target selectivity, low side-effects and high efficiency, which are suitable for the treatment of abnormal expression of genes in various diseases (8). Importantly, this technology can be used to prevent changes in tumor-control genes at the nucleic acid level.

Livin is specifically over-expressed in tumor cells, but not in normal tissues in adults, suggesting that it could be a novel target that induces tumor cell apoptosis. RNA interference technology has been extensively used to silence gene expression in vitro and in vivo. Using RNAi strategy, Crnkovic et al (9) silenced endogenous Livin expression in HeLa cells and found that the apoptosis rate had increased significantly after treatment with doxorubicin, UV irradiation, and tumor necrosis factor $\alpha$, indicating that Livin could become a molecular target for gene therapy of cancer. Livin expression was dramatically decreased in HCT116 colon cancer cells after siRNA transfection, and xenografted tumor size in nude mice had significantly decreased after Livin siRNA injection at a concentration of $20 \mu \mathrm{mol} / \mathrm{L}$ (10). These studies suggested that inhibiting Livin expression could serve as a potential tumor-specific therapeutic strategy.

In this study, we designed the shRNA targeting the coding sequence of Livin- $\alpha$, a splice variant of Livin, and inserted the single-stranded Livin fragments after annealing into a vector carrying EGFP and U6 promoter. The Livin shRNA expression vector was successfully constructed and transfected into laryngeal carcinoma Hep-2 cells. After screening, the stably transfected Hep-2 cells could transcribe a large number of shRNAs that inhibited Livin expression. Quantitative real-time PCR showed that the relative expression of Livin mRNA in cells transfected with the positive plasmid was $0.53 \pm 0.02$, which was significantly lower as compared to the cells transfected with the negative control plas- mid $(100 \%)$, and the inhibition rate was $47.17 \%$. Western blotting results showed that the relative expression of Livin protein in cells transfected with the positive plasmid was $0.48 \pm 0.03$, which was significantly lower as compared to the cells transfected with the negative control plasmid $(0.73 \pm 0.03)$, and the inhibition rate was $34.25 \%$, which was consistent with the results of quantitative real-time PCR. Our findings confirmed that the pGenesil-LivinshRNA recombinant plasmid could reduce the expression of Livin at the mRNA as well as protein levels. Therefore, our study has laid a strong foundation for future biological research of laryngeal carcinoma cells using Livin gene as a target, and provided an experimental system for gene therapy of cancer.

\section{References}

1. Vucic D, Stennicke HR, Pisabarro MT et al. ML-IAP, a novel inhibitor of apoptosis that is preferentially expressed in human melanomas. Curr Biol 2000; 10: 1359-1366.

2. Wang X, Xu J, Ju S et al. Livin gene plays a role in drug resistance of colon cancer cells. Clin Biochem 2010; 43: 655-660.

3. Choi J, Hwang YK, Sung KW et al. Expression of Livin, an antiapoptotic protein, is an independent favorable prognostic factor in childhood acute lymphoblastic leukemia. Blood 2007; 109: 471-477.

4. Gazzaniga P, Gradilone A, Giuliani L et al. Expression and prognostic significance of Livin, survivin and other apoptosisrelated genes in the progression of superficial bladder cancer. Ann Oncol 2003; 14: 85-90.

5. Hariu H, Hirohashi Y, Torigoe T et al. Aberrant expression and potency as a cancer immunotherapy target of inhibitor of apoptosis protein family, Livin/ML-IAP in lung cancer. Clin Cancer Res 2005; 11: 1000-1009.

6. Xiang Y, Yao H, Wang S et al. Prognostic value of survivin and livin in nasopharyngeal carcinoma. Laryngoscope 2009; 116: 126-130.

7. Sashital DG, Doudna JA. Structural insights into RNA interference. Curr Opin Struct Biol 2010; 20: 90-97.

8. Kalota A, Dondeti VR, Gewirtz AM et al. Progress in the development of mucleic acid therapeutics. Handb Exp Pharmacol 2006; 173: 173-196.

9. Crnkovic-Mertens I, Semzow J, Hoppe-Seyler F et al. Isoform-specific silencing of the Livin gene by RNA interference defines Livin beta as key mediator of apoptosis inhibition in HeLa cells. J Mol Med 2006; 84: 232-240.

10. BO-Young Oh, Ryung-AhLee, Kwang Ho Kim. SiRNA targeting livin decreases tumor in a xenograft model for colon cancer. World J Gastroenterol 2011; 17: 2563-2571. 